\title{
Human Dirofilariasis: A Case of Infraorbital Infection
}

\section{Shankar $\mathrm{A}^{1 *}$ and Samuel $\mathrm{P}^{2}$}

1Department of ENT, Cosmopolitan Hospital \& Research Centre, India

${ }^{2}$ Department of Otorhinolaryngology, Cosmopolitan Hospital \& Research Centre, India

*Corresponding author: Abhijit Shankar, Department of ENT, Cosmopolitan Hospital \& Research Centre, Pattom, Thiruvananthapuram- 695004, Kerala, India, Email: drabhijitshankar@gmail.com

\section{Case Report}

Volume 6 Issue 2

Received Date: August 16, 2021

Published Date: August 31, 2021

DOI: $10.23880 /$ ooaj- 16000220

\section{Abstract}

Background: Human dirofilariasis is not perceived as a serious public health concern in India despite more number of sporadic cases being reported of late. Only a few dozen cases were reported in the first decade of the new millenium, but the number is gradually increasing during the last decade. With a high population of pet dogs \& cats and vast number of stray dogs, the State of Kerala could be a potential infective area.

Case Report: It is against this background that an interesting case of infraorbital incidence of dirofilaria repens is being reported that was mimicking as periorbital cellulitis. The cyst was surgically excised and sent for histopathological examination. The presence of Dirofilaria repens was confirmed.

Conclusion: There has been an increase in the incidence of dirofilariasis in countries that were considered as non-endemic such as India of late. The probable contributing factors are discussed in detail. Coordinated parasite surveillance and preventive vector control measures with active participation from LSGs and local population are suggested.

Keywords: Dirofilaria; Zoonotic Filariasis; Pet Associated Health Hazard; Mosquito Vectors

\section{Introduction}

Zoonotic filariasis, i.e., human infection with filaria of animals, occur worldwide. The zoonotic filariae recovered commonly from humans belong to the genus Dirofilaria [1]. Dirofilaria is a filarial nematode that infects mostly mammals like dogs, other canines like wolves, foxes and cats. Out of forty species of Dirofilaria identified, D. repens and $D$. immitis have been most commonly associated with human infections. The human beings are accidental hosts [1-3]. The infecting larva is $\mathrm{L}_{3}$ or the filariform larva of third stage [4]. Mosquitoes belonging to the genera of Anopheles, Culex, Armigeresor Aedes can act as vectors for transmission [4].

D. immitis is a parasite that commonly infects cardiovascular system of canines with a potential to involve human pulmonary system. D. repens commonly localizes in the subcutaneous tissue of definitive host. In humans, the infection usually presents itself as solitary nodule, but the worms may migrate to the subcutaneous tissue causing creeping eruptions. The clinical form of dirofilariasis in humans is pulmonary, subcutaneous, ocular, and rarely infection of the cerebrum, testicles, and female breasts, invariably leading to incorrect diagnosis of malignant tumour $[4,5]$. Ocular involvements have been reported as orbital, periorbital, subconjunctival or intravitreal [4]. There are reports where conjunctival dirofilariasis has been misdiagnosed as allergic conjunctivitis [5]. We report a case of Dirofilaria recovered from the right infraorbital tissue of a 48 year old female patient, who was presented with complaint of swelling in her right eye. 


\section{Case Report}

A 48 year old female presented in the outpatient wing of the Otorhinolaryngology department of our institution with presenting complaints of painful swelling just beneath her right eye for a duration of past 2 days (Figure 1). History elicitation revealed that she had a mild discomfort with occasional puffiness of her lower eyelid for the past 6 months. She had been suffering from hypothyroidism for the past 14 years and was on thyroxine supplementation. She was seeking treatment at various hospitals, with only mild relief to her distress. She was referred from a private health care centre specializing in eye care with inconclusive diagnosis of periorbital cellulitis.

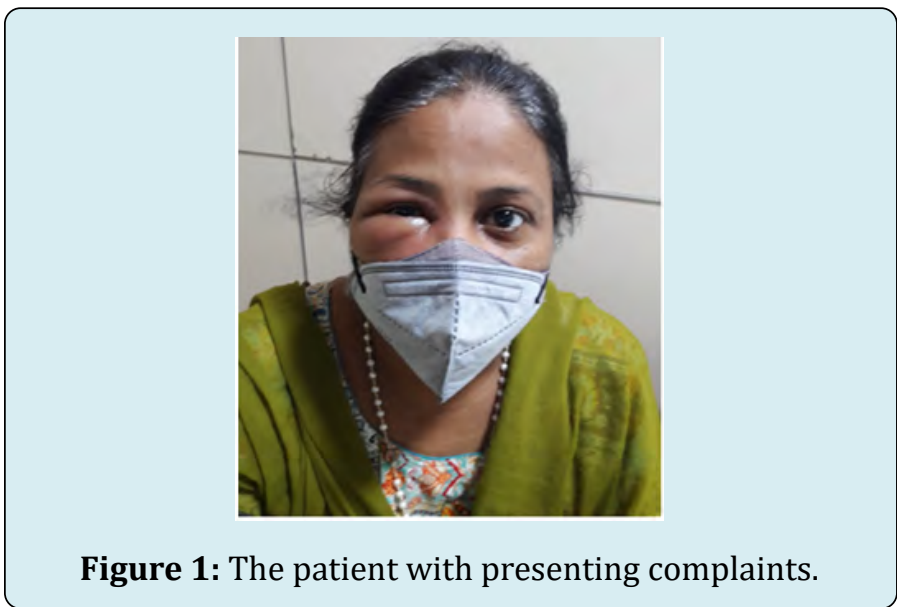

Plain CT imaging of the orbit and paranasal sinus indicated a cystic area within the soft tissue planes of right premaxillary region with subtle internal cystic density (Figure 2). An Ultrasonography (USG) was done in order to correlating the findings. It showed a heterogeneously hyperechoic ovoid space occupying lesion with a tiny linear component showing coiled orientation that appeared mobile within the cyst. No definitive fluid collection was evident except surrounding areas of cellulitis and fluid tracking. Both lacrimal glands and optic nerve sheath complex were normal. Imaging features strongly pointed towards the possibility of complicated premaxillary parasite cyst with consequent preseptal cellulitis (Figure 3). In order to have clinico-pathologic correlation, the cyst was surgically excised and sent for histopathological examination. She was put on albendazole oral steroids and other supportives on being sent home for follow up. The histopathological evaluation revealed fibromuscular tissue with dense infiltration by eosinophils and lymphoplasmacytes. Typical cuticle of Dirofilaria repens was also identified (Figure 4), leading to the presence of Dirofilaria repens. On cross-checking, the presence of pet dogs and cats were confirmed at the patient's residence. Clinical follow up after one week showed complete recovery (Figure 5) with full disappearance of the presenting symptoms.

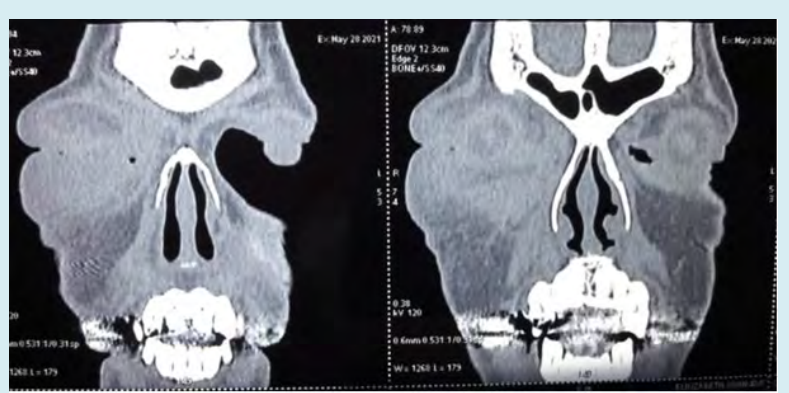

Figure 2: CT Paranasal sinus and orbit.

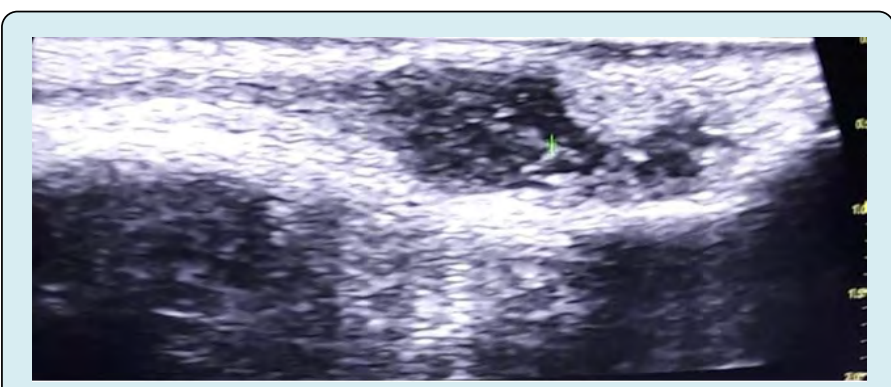

Figure 3: USG view of encysted worm.

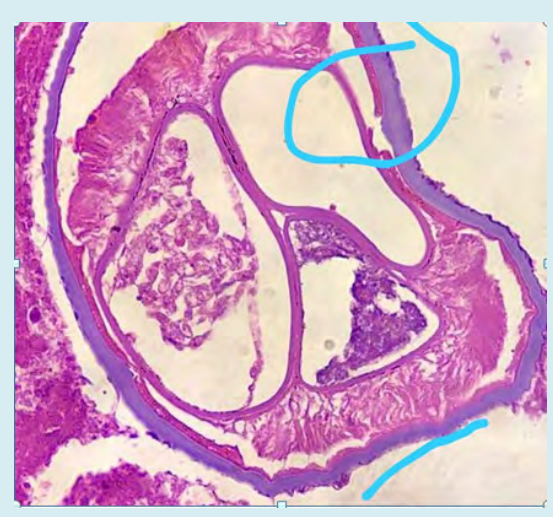

Figure 4: Microscopic cross sectional view of the worm.

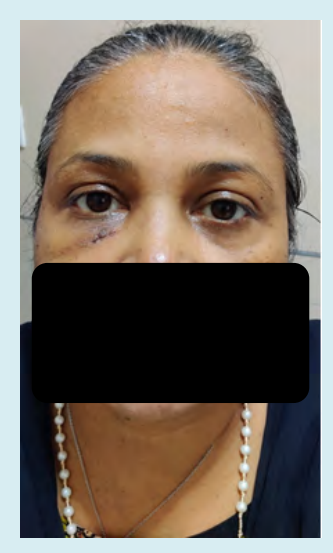

Figure 5: The patient on follow up visit. 


\section{Otolaryngology Open Access Journal}

\section{Discussion}

Cases of dirofilaria were sporadically reported in India, which is considered to be a non-endemic area $[5,6]$. The diagnosis of human infection by Dirofilaria repens poses many challenges. In many cases, the presenting symptoms can vary and non-specific, depending upon the location of the worm [4]. It includes transitory inflammatory swellings or nodules that may or may not be painful. Acute symptoms appear when the worms enter the conjunctiva, which compels the patient to seek medical attention. It should be noted that the $\mathrm{L}_{3}$ infective larva of the size of about one mm requires 6 to 8 months to develop into an adult with a size of $10 \mathrm{~cm}$ or more [4]. Hence, the migration of the worm through sensitive tissues, especially in the head region could lead to tremendous discomfort, irritation or inflammation. Kalogeropoulos, et al. [5] have reported that in suspected cases of dirofilaria, blood sample analysis showed an increase in the number of eosinophils, which returns to the normal levels after the removal of the parasite.

\section{Conclusion}

Recent reports suggest that there has been an increase in the incidence of dirofilariasis in countries that were considered as non-endemic such as India [5]. With a high population of pet dogs \& cats and vast number of stray dogs, the State of Kerala could be a potential infective area, especially in view of the fact that rearing of pet dogs and cats do not require any registration or licencing from the local self-governments (LSGs). The situation is made grimmer by the abundance of Culex, Anopheles and Aedes mosquito population with a prolonged warm, humid summer. An increasing influx of emigrant labour force from the State, who travel frequently to endemic areas and a large influx of tourists from endemic areas like Europe (Italy, France, Greece, Croatia, Serbia, Denmark and Russia), African countries like Tunisia and back to tourism destinations in Kerala are to be considered in this context. As a result, more cases could be reported in future and more awareness about dirofilariasis is called for before it becomes a serious public health concern. Thrust on a coordinated and systematic parasite surveillance to put the canine reservoir under check and preventive vector (mosquito) control measures with active participation from LSGs and local population assumes vital importance in arresting rapid transmission.

\section{References}

1. Orihel TC, Eberhard ML (1998) Zoonotic filariasis. Clin Microbiol Rev 11(2): 366-381.

2. Nath R, Gagoi R, Bardoloi N, Gogoi T (2010) Ocular Dirofilariasis. Indian J Pathol Microbiol 53(1): 157-159.

3. Mahajan RK, Sharma S, Das S, Malik PK (2017) Human Ocular Dirofilariasis- An Emerging Zoonotic Infection in India. JoJ Case Stud 5(3): 1-3.

4. Sabu L, Devada K, Subrammanian H (2005) Dirofilariasis in dogs and humans in Kerala. Indian J Med Res 121(5): 691-693.

5. Kalogeropoulos CD, Stefaniotou MI, Gorgoli KE, Papadopoulo CV, Pappa CN, et al. (2014) Ocular Dirofilariasis: A case series of 8 patients. Middle East Afr J Opthalmol 21(4): 312-316.

6. Chopra R, Bhatti SM, Mohan S, Taneja N (2012) Dirofilaria in the anterior chamber: A rare occurrence. Middle East Afr J Opthalmol 19(3): 349-351. 